\title{
Anti-apoptotic effects of suppressor of cytokine signaling 3 and 1 in psoriasis
}

\author{
S Madonna ${ }^{\star, 1,3}$, C Scarponi ${ }^{1,3}$, S Pallotta ${ }^{2}$, A Cavani $^{1}$ and C Albanesi ${ }^{1}$
}

Because of their genetically determined capacity to respond to pro-inflammatory stimuli, keratinocytes have a crucial role in the pathogenesis of psoriasis. Upon IFN- $\gamma$ and TNF- $\alpha$ exposure, psoriatic keratinocytes express exaggerated levels of inflammatory mediators, and show aberrant hyperproliferation and terminal differentiation. The thickening of psoriasic skin also results from a peculiar resistance of keratinocytes to cytokine-induced apoptosis. In this study, we investigated on the molecular mechanisms concurring to the resistance of psoriatic keratinocytes to cell death, focusing on the role having suppressor of cytokine signaling (SOCS)1 and SOCS3, two molecules abundantly expressed in IFN- $\gamma /$ TNF- $\alpha$-activated psoriatic keratinocytes, in sustaining antiapoptotic pathways. We found that SOCS1 and SOCS3 suppress cytokine-induced apoptosis by sustaining the activation of the PI3K/AKT pathway in keratinocytes. The latter determines the activation of the anti-apoptotic NF- $\kappa$ B cascade and, in parallel, the inhibition of the pro-apoptotic BAD function in keratinocytes. For the first time, we report that phosphorylated AKT and phosphorylated BAD are strongly expressed in lesional psoriatic skin, compared with healthy or not lesional skin, and they strictly correlate to the high expression of SOCS1 and SOCS3 molecules in the psoriatic epidermis. Finally, the depletion of SOCS1 and SOCS3, as well as the chemical inactivation of PI3K activity in psoriatic keratinocytes, definitively unveils the role of PI3K/AKT cascade on the resistance of diseased keratinocytes to apoptosis.

Cell Death and Disease (2012) 3, e334; doi:10.1038/cddis.2012.69; published online 28 June 2012

Subject Category: Immunity

Suppressor of cytokine signaling (SOCS) is a family of eight intracellular proteins (cytokine-inducible Src homology 2-containing protein and SOCS1 to SOCS7), which act as endogenous inhibitors of pro-inflammatory pathways triggered by various cytokines. ${ }^{1,2}$ Among these, SOCS1 negatively regulates IFN- $\gamma$ signaling by binding and inactivating Jak2 protein and, in turn, impeding the activation of STAT1 transcription factor. ${ }^{3}$ The inhibitory effect of SOCS1 on IFN- $\gamma$ signaling has been unequivocally demonstrated in human keratinocytes, where the ectopic SOCS1 overexpression determines a reduced production of IFN- $\gamma$-dependent genes. $^{4-7}$ In addition, SOCS1 is involved in the TNF- $\alpha$ signaling, as it inhibits TNF- $\alpha$-induced NF- $\kappa$ B cascade by decreasing p65 stability within the cell nucleus as well as ASK1/JNK pathway. ${ }^{8,9}$ SOCS3 is another important negative regulator of cytokine signaling, and it is known to suppress IL-6 and G-CSF cascades, by directly binding to the specific receptors. $^{10,11}$ SOCS1 and SOCS3 act not only as antiinflammatory checkpoints but also as important protective and pro-survival molecules in various cell types. In particular, SOCS1 can repress apoptosis induced by TNF- $\alpha$ by sustaining the activation of p38 MAP kinase signaling in murine fibroblasts or by inhibiting JAK activation in Jurkat T-cell line, ${ }^{12,13}$ whereas SOCS3 has been reported to inhibit TNF- $\alpha$-induced apoptosis in pancreatic beta cells by impairing the activation of ERK1/2, p38 and JNK pathways. ${ }^{14}$ SOCS3 also suppresses apoptosis in cancer cells, as demonstrated in renal carcinoma cells undergoing IFN- $\alpha$-induced apoptosis. ${ }^{15}$

Apoptosis is a form of programmed cell death mediated by different stimuli, including binding of TNF- $\alpha$ to death TNFR1 receptor. In the continuously renewing skin tissue, mechanisms of proliferation, differentiation and cell death are tightly regulated to prevent inappropriate and excessive epidermal growth. ${ }^{16,17}$ However, dysfunctional apoptosis can occur in the skin, and it determines the development and chronicization of several skin diseases, in particular in psoriasis. In this pathological context, epidermal keratinocytes are characterized by an aberrant resistance to apoptosis, which concurs to the peculiar thickening of psoriatic epidermis. ${ }^{18}$ Multiple factors have been proposed to contribute to the reduced susceptibility of keratinocytes to apoptosis, such as their predisposition to express enhanced levels of the antiapoptotic molecules survivin and BCL- $\mathrm{X}_{\mathrm{L}}{ }^{19,20}$

In this study, we investigated on the molecular mechanisms involved in rendering psoriatic keratinocytes resistant to cell death, focusing on the role having SOCS1 and SOCS3 molecules in this context. Firstly, we demonstrated that SOCS1 and SOCS3 molecules, both abundantly expressed

\footnotetext{
${ }^{1}$ Laboratory of Experimental Immunology, Istituto Dermopatico dell'Immacolata (IDI)-IRCCS, Rome, Italy and ${ }^{2} \mathrm{~V}$ Division of Dermatology, Istituto Dermopatico dell'Immacolata (IDI)-IRCCS, Rome, Italy

*Corresponding author: S Madonna, Laboratory of Experimental Immunology, Istituto Dermopatico dell'Immacolata (IDI)-IRCCS, Via Monti di Creta, 104, 00167 Rome, Italy. Tel: + 3906 66464776; Fax: + 3906 66464705; E-mail: madonnastefania@ gmail.com

${ }^{3}$ These authors equally contributed to this work.

Keywords: keratinocyte apoptosis; psoriasis; SOCS molecules; pro-survival AKT pathway

Abbreviations: SOCS1/3, suppressor of cytokine signaling 1/3; Abs, antibodies; IFN- $\gamma$, interferon-gamma; TNF- $\alpha$, tumor necrosis factor- $\alpha$; siRNA, small interfering RNA; PI3K, phosphoinositide 3-kinase; JAK2, Janus kinase 2; BCL-XL, B-cell lymphoma-extra large; UV, ultraviolet

Received 07.3.12; revised 27.4.12; accepted 08.5.12; Edited by E Candi
} 
in psoriatic epidermis, suppress the IFN- $\gamma / T N F-\alpha$-induced apoptosis in human keratinocytes by specifically sustaining the activation of the anti-apoptotic PI3K/AKT pathway. Moreover, we showed that PI3K/AKT activation drives survival programs in cytokine-activated keratinocytes, by triggering the anti-apoptotic NF- $\kappa \mathrm{B}$ cascade and, in parallel, by inhibiting the pro-apoptotic BAD molecule. For the first time, in this study we documented that phosphorylated AKT and phosphorylated BAD are strongly expressed in lesional psoriatic skin, compared with healthy or not lesional skin, and they strictly correlate to the expression of SOCS molecules in the psoriatic epidermis. Finally, the depletion of SOCS1 and SOCS3, similar to the chemical inhibition of PI3K/AKT pathway in psoriatic keratinocytes, reverts the reduced susceptibility of these cells to cytokine-induced apoptosis, definitively confirming the influence of this molecular cascade on the peculiar resistance of diseased keratinocytes to cell death.

\section{Results}

Psoriatic keratinocytes are more predisposed than healthy cells to express SOCS3 and SOCS1 in response to IFN- $\gamma /$ TNF- $\alpha$ stimulation. To compare SOCS levels in healthy and psoriatic keratinocyte strains, we isolated cells from skin biopsies obtained from psoriatic patients and healthy donors, and left untreated or stimulated with IFN- $\gamma$ plus TNF- $\alpha$ for $3 \mathrm{~h}$. We found that SOCS3 and SOCS1 mRNA and protein expression was substantially higher in IFN- $\gamma /$ TNF- $\alpha$-activated keratinocytes obtained from psoriatic patients compared with those isolated from healthy donors (Figure $1 \mathrm{a}, P<0.01$ and $P<0.05$ for SOCS3 and SOCS1, a

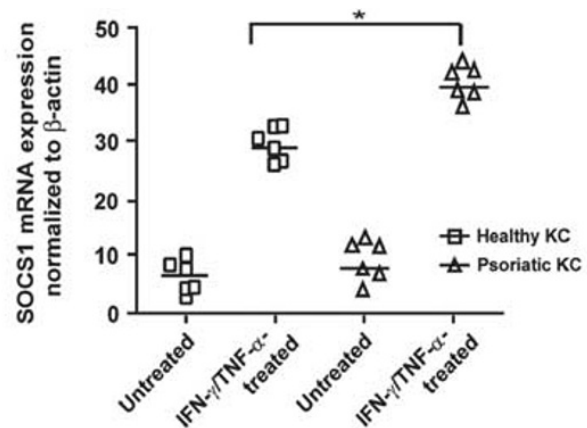

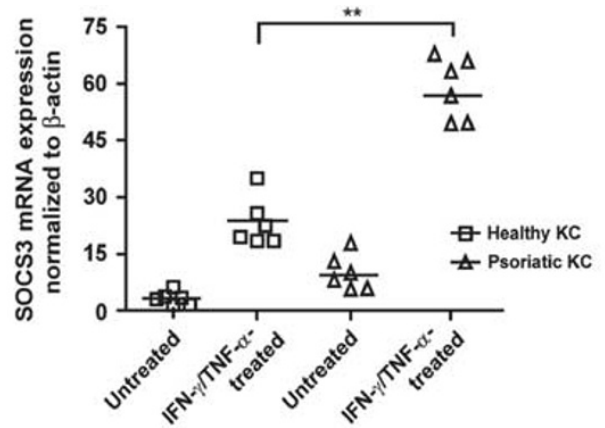

b
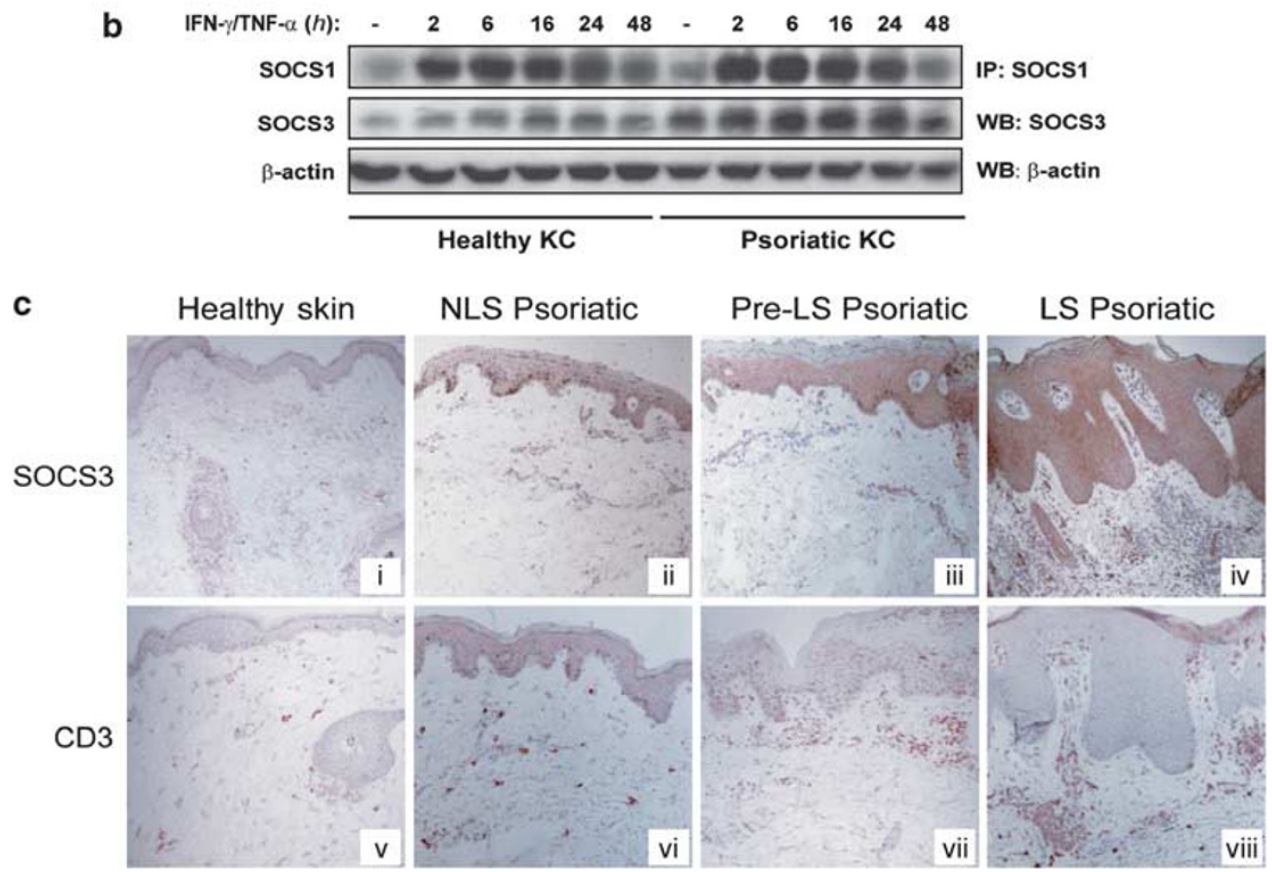

Figure 1 IFN- $\gamma / T N F-\alpha$-induced SOCS1 and SOCS3 expression is higher in psoriatic keratinocytes than in healthy cells. (a) Cultured keratinocytes were prepared from healthy skin or from biopsies taken from uninvolved psoriatic skin. SOCS1 and SOCS3 mRNA levels were detected by real-time PCR analysis in cultured healthy (Healthy KC, $\square)(n=6)$ and psoriatic keratinocytes (PS KC, $\Delta)(n=6)$ left untreated or $3 \mathrm{~h}$ stimulated with IFN- $\gamma$ and TNF- $\alpha$ and normalized with $\beta$-actin mRNA values. Horizontal lines indicate mean values for each experimental group. (b) Protein extracts obtained from healthy $(n=6)$ and psoriatic $(n=6)$ keratinocytes, pre-treated with MG132 for $2 \mathrm{~h}$ and, then, stimulated with IFN- $\gamma$ and TNF- $\alpha$ for different time periods, were subjected to immunoprecipitation for SOCS1 and western blotting analysis to detect SOCS3 or SOCS1 expression. One representative healthy $\mathrm{KC}$ and psoriatic KC strain is shown. (c) Immunohistochemistry for SOCS3 and CD3 (both stained in red) was performed on cryosections from biopsies of healthy skin ( $n=4$ ) (i and v) and psoriatic skin $(n=4)$ including NLS (ii and vi), proximal-to-lesion (Pre-LS) (iii and vii) and LS (iv and viii) zones of evolving plaques. Sections were counterstained with Mayer's H\&E.

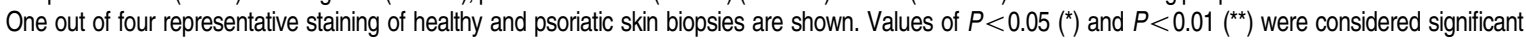


respectively; Figure 1b). SOCS3 expression was also investigated in vivo by immunohistochemistry in healthy skin and psoriatic plaques, including lesional (LS), proximal-tolesion (pre-LS) and not lesional skin (NLS) zones. As shown in Figure 1c, SOCS3 staining was intense throughout the epidermis of LS psoriasis, with a predominant localization in the spinosum and granulosum layers (panel iv), and correlated to the presence in the upper dermis of infiltrating $\mathrm{CD}^{+} \mathrm{T}$ lymphocytes (panel viii). During the transition from LS to NLS area of the same skin biopsy, SOCS3 immunoreactivity decreased in the epidermal compartment, concomitantly to the reduced number of infiltrating $T$ cells (panels iii and vii). Consistently, SOCS3 was not present in healthy skin and in NLS psoriatic skin (panels $\mathrm{i}$ and ii), where a very low number of $\mathrm{CD}^{+}{ }^{+} \mathrm{T}$ cells in the dermis were present (panels $v$ and vi).

SOCS3 and SOCS1 suppress cytokine-induced apoptosis in human keratinocytes. SOCS1 and SOCS3 have been described as anti-inflammatory molecules able also to suppress apoptosis induced by different stimuli. ${ }^{1,12,14}$ To investigate whether SOCS1 and/or SOCS3 can protect human keratinocytes from cytokine-induced cell death, we treated Myc-SOCS1 or -SOCS3-overexpressing HaCaT clones for $48 \mathrm{~h}$ with TNF- $\alpha$, a potent cytokine inducer of keratinocyte apoptosis, and examined their apoptosis rate by measuring annexin staining and $\mathrm{PI}$ incorporation (Figure 2a). SOCS2 clones were analyzed to determine whether other SOCS family members provided resistance against TNF- $\alpha$ induced cell death. Contrarily to SOCS2, SOCS1 $(P<0.05)$ and, more significantly, SOCS3 clones $(P<0.01)$ showed an enhanced resistance to TNF- $\alpha$-induced apoptosis compared with MOCK clones (Figure 2a). Also the expression of active caspase 9 , a mediator of apoptosis triggered by TNF- $\alpha$, was reduced in SOCS3 and SOCS1 compared with activated MOCK or SOCS2 clones (Figure 2a). As expected, SOCS1and, more efficiently, SOCS3-silenced keratinocytes resulted to be more susceptible to IFN- $\gamma /$ TNF- $\alpha$-induced apoptosis compared with cells transfected with irrelevant siRNA (Figure $2 \mathrm{~b}, P<0.01$ for SOCS3, and $P<0.05$ for SOCS1). Taken together, these findings reveal that SOCS1 and, more efficiently, SOCS3 protect human keratinocytes from cytokine-induced death.

SOCS3 and SOCS1 sustain RAS/AKT cascade, which counteracts cytokine-induced apoptosis in keratinocytes. SOCS1 contributes to the induction of RAS and downstream ERK1/2, a pro-survival cascade protecting keratinocytes from the detrimental effects of pro-inflammatory cytokines. ${ }^{5}$ As the anti-apoptotic PI3K/AKT pathway is also a crucial effector of RAS, ${ }^{21,22}$ we analyzed whether SOCS1 and SOCS3 could sustain AKT activation, and whether this activation, in turn, could protect keratinocytes from the TNF- $\alpha$-induced apoptosis. Firstly, contrarily to MOCK and SOCS2 clones, SOCS1 and SOCS3 strains showed a relevant basal activation of RAS, while it was drastically reduced in SOCS3- and in SOCS1-silenced cells upon IFN- $\gamma /$ TNF- $\alpha$-treatment (Figure $3 a, P<0.01$ for SOCS3 and $P<0.05$ for SOCS1), as compared with control keratinocytes. In addition, SOCS1 and, more efficiently,
SOCS3 supported AKT phosphorylation in serine 473, as demonstrated in SOCS-overexpressing clones and in SOCS3- or SOCS1-depleted keratinocytes treated with IFN- $\gamma$ and TNF- $\alpha$ (Figure $3 b$ ). In a next series of experiments, we showed that, when treated with increasing doses of the chemical PI3K inhibitor, Ly29, the susceptibility of SOCS1 and SOCS3 clones to TNF- $\alpha$-induced death increased, differently from what was observed for MOCK or SOCS2 cells (Figure 4a). Ly29 effect reverting the anti-apoptotic SOCS1 and SOCS3 function was specific, as other chemical inhibitors, such as the JAK2 inhibitor AG490 or the ERK1/2 inhibitor PD98059, had null or only slight effect on TNF- $\alpha$ induced apoptosis (Figure 4b). Therefore, PI3K/AKT pathway sustained by SOCS3 and SOCS1 specifically protects keratinocytes from cytokine-induced death.

AKT activation by IFN- $-\gamma /$ TNF- $\alpha$ triggers NF- $\kappa$ B cascade and, in parallel, induces phosphorylation and inactivation of BAD in human keratinocytes. AKT activation occurs in IFN- $\gamma /$ TNF- $\alpha$-treated keratinocytes with delayed kinetics, being its phosphorylation substantially upregulated 12-24 $\mathrm{h}$ after stimulation (Figure 5a). As human keratinocytes exposed to UV radiation show protective AKT-mediated programs involving $\mathrm{NF}-\kappa \mathrm{B}$ and the pro-apoptotic $\mathrm{BAD}$ molecule, ${ }^{23,24}$ we analyzed the NF- $\kappa \mathrm{B}$ and $\mathrm{BAD}$ cascades in keratinocyte cultures pre-treated with Ly29 and stimulated with IFN- $\gamma$ plus TNF- $\alpha$. AKT inhibition determined a massive accumulation of $\mathrm{I}_{\kappa} \mathrm{B} \alpha$ protein, the endogenous inhibitor of NF$\kappa \mathrm{B}$ family, and the decrease of the phosphorylated p65 (Ser276) factor (Figure 5b). As a possible consequence of NF$\kappa \mathrm{B}$ downregulation by $\mathrm{PI} 3 \mathrm{~K} / \mathrm{AKT}$ inhibition, we found a quite totally compromised expression of the anti-apoptotic BCL-2 protein, a well-known NF- $\kappa \mathrm{B}$ transcriptional target $^{25}$ (Figure $5 b$ ). Contrarily, the expression of $B C L-X_{L}$, another pro-survival molecule known to be activated by NF- $\kappa \mathrm{B}$ pathway, was not affected by the inhibition of AKT activity. However, BCL- $X_{L}$ fraction associated to the pro-apoptotic p-BAD molecule, barely detectable in cells not treated with Ly29, increased following Ly29 treatment, concomitantly to a significant reduction of phosphorylated BAD, an inactive form of BAD (Figure $5 b$ ). Therefore, AKT activation by IFN- $\gamma /$ TNF- $\alpha$ in human keratinocytes executes anti-apoptotic functions by activating the pro-survival NF- $\kappa \mathrm{B}$ cascade and, in parallel, by inducing $B A D$ phosphorylation/inactivation and dissociation from the anti-apoptotic BCL- $X_{L}$ molecule.

Phospho-AKT, phospho-BAD and BCL- $X_{L}$ are strongly expressed in activated psoriatic keratinocytes. The hyperplasia of the psoriatic epidermis is in part due to a peculiar resistance of psoriatic keratinocytes to apoptotic stimuli compared with healthy cells. ${ }^{26-29}$ As we showed that AKT signaling can trigger survival signals in IFN- $\gamma /$ TNF- $\alpha$ activated keratinocytes, we sought to analyze whether phosphorylated AKT and downstream phosphorylated p65, phosphorylated BAD and BCL- $\mathrm{X}_{\mathrm{L}}$ anti-apoptotic molecules could be upregulated in psoriatic skin in vivo and in keratinocyte strains isolated from skin of psoriatic patients. Phospho-AKT (Ser473) staining was intense in the spinosum and granulosum layers of LS psoriatic epidermis, whereas it was quite absent in NLS area of the same biopsies and in 

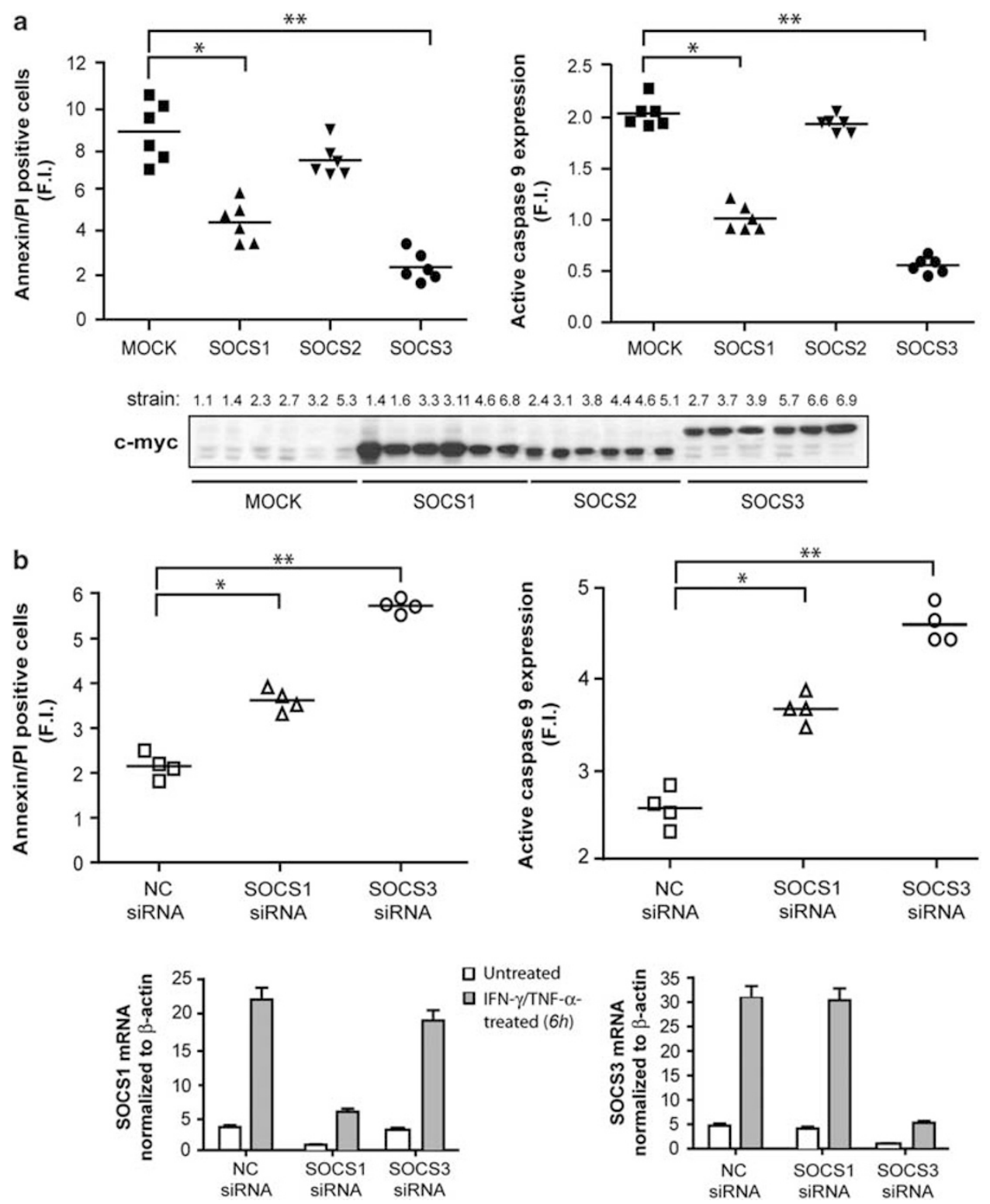

Figure 2 Cytokine-induced apoptosis in human keratinocytes is suppressed by SOCS1 and SOCS3 molecules. (a) HaCaT cells were stably transfected with myc-tagged socs1, socs2 and socs3 genes or control plasmids. MOCK $(\boldsymbol{\nabla}, n=6)$, SOCS1 $(\boldsymbol{\Lambda}, n=6), \operatorname{SOCS} 2(\boldsymbol{\nabla}, n=6)$ and SOCS3 $(\bullet, n=6)$ clones were left untreated or stimulated with TNF- $\alpha$ for $48 \mathrm{~h}$ and their apoptosis was examined by measuring Annexin/PI fluorescent staining or expression of active caspase 9 through FACS analysis. Data were expressed as F.I. between TNF- $\alpha$-treated samples and untreated samples, to which a value of 1 was given. Ectopic SOCS1, SOCS2 and SOCS3 expression in different clones was determined by immunoblotting with an anti-c-myc Ab. (b) Transient RNA interference was performed by transfecting cultured healthy keratinocytes with SOCS1, SOCS3 or irrelevant (NC) siRNA, and then by treating cells with IFN- $\gamma / T N F-\alpha$ for $48 \mathrm{~h}$. Apoptosis was evaluated as described in (a). SOCS1 and SOCS3 mRNA levels in siRNA-transfected cells were measured by real-time PCR. SOCS3 mRNA levels were monitored to validate the specificity of SOCS1 siRNA, and vice versa. All data were obtained from three independent experiments of RNA interference. Values of $P<0.05\left(^{*}\right)$ and $P<0.01\left(^{* *}\right)$ were considered significant

healthy epidermis (Figure 6a). Also phospho-p65, phospho $B A D$ and $B C L-X_{L}$ expression was higher compared with healthy and NLS psoriatic skin, and mainly localized in the upper layers of the psoriatic epidermis (Figure 6a). Consistently with the in vivo analysis, cultured psoriatic strains were more prone than healthy cells to express enhanced levels of phospho-AKT in response to cytokine treatment (Figure 6b). Therefore, AKT staining in psoriatic epidermis fits with the downstream anti-apoptotic phosphop65, phospho-BAD and BCL- $X_{L}$ molecules, in terms not only of expression levels but also of localization within the psoriatic epidermal compartment.

SOCS3 and SOCS1 overexpression in activated psoriatic keratinocytes contributes to their peculiar resistance to cytokine-induced apoptosis. Finally, we investigated whether SOCS3 and SOCS1 participated to the reduced sensitivity of psoriatic keratinocytes to cytokineinduced death. Firstly, we assessed that the apoptotic rate of cultured psoriatic keratinocytes in response to IFN- $\gamma$ and 
a
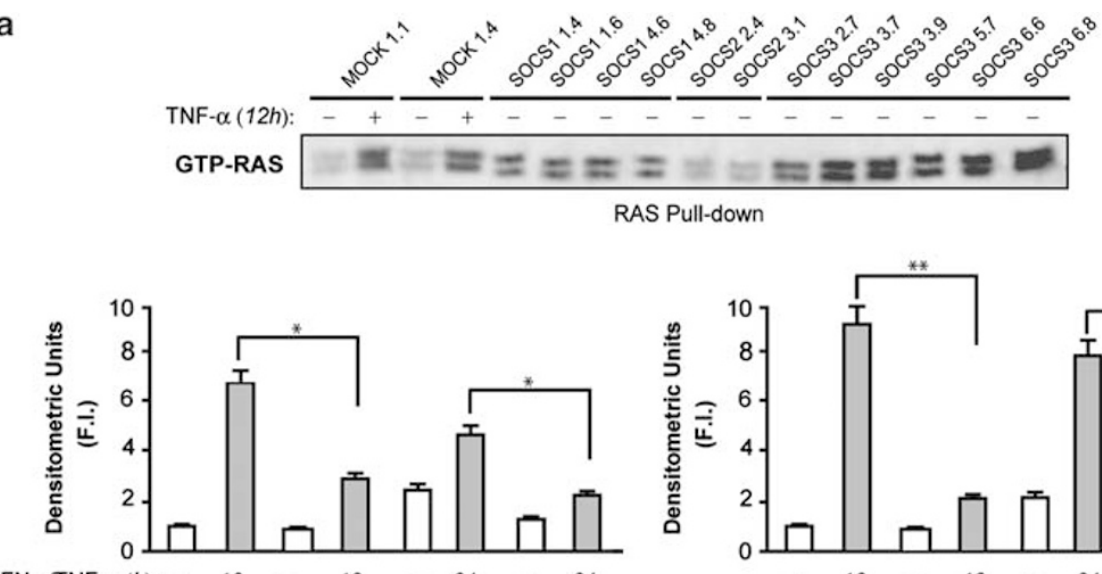

IFN- $\gamma /$ TNF- $\alpha(h):-12-12-24$ - 24

GTP-RAS
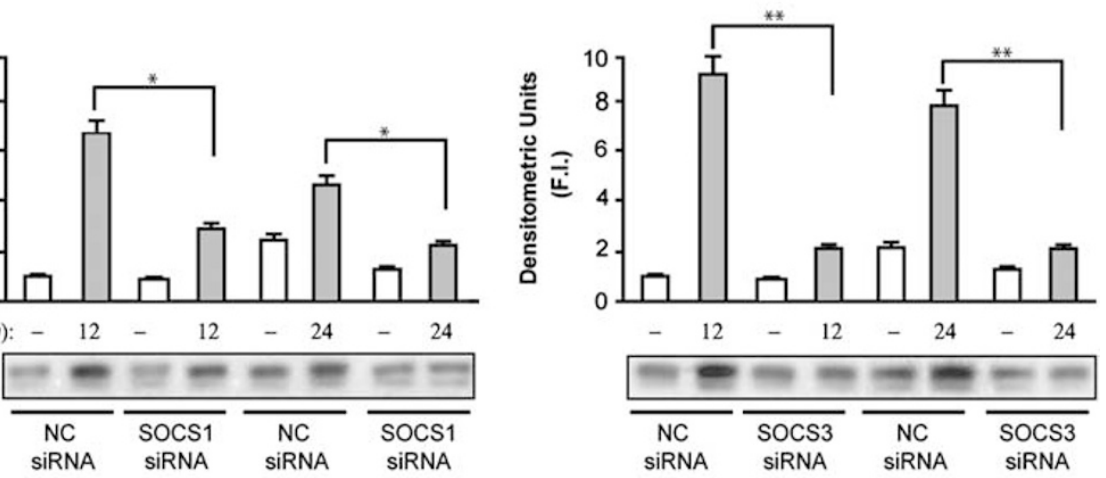

RAS Pull-down

b
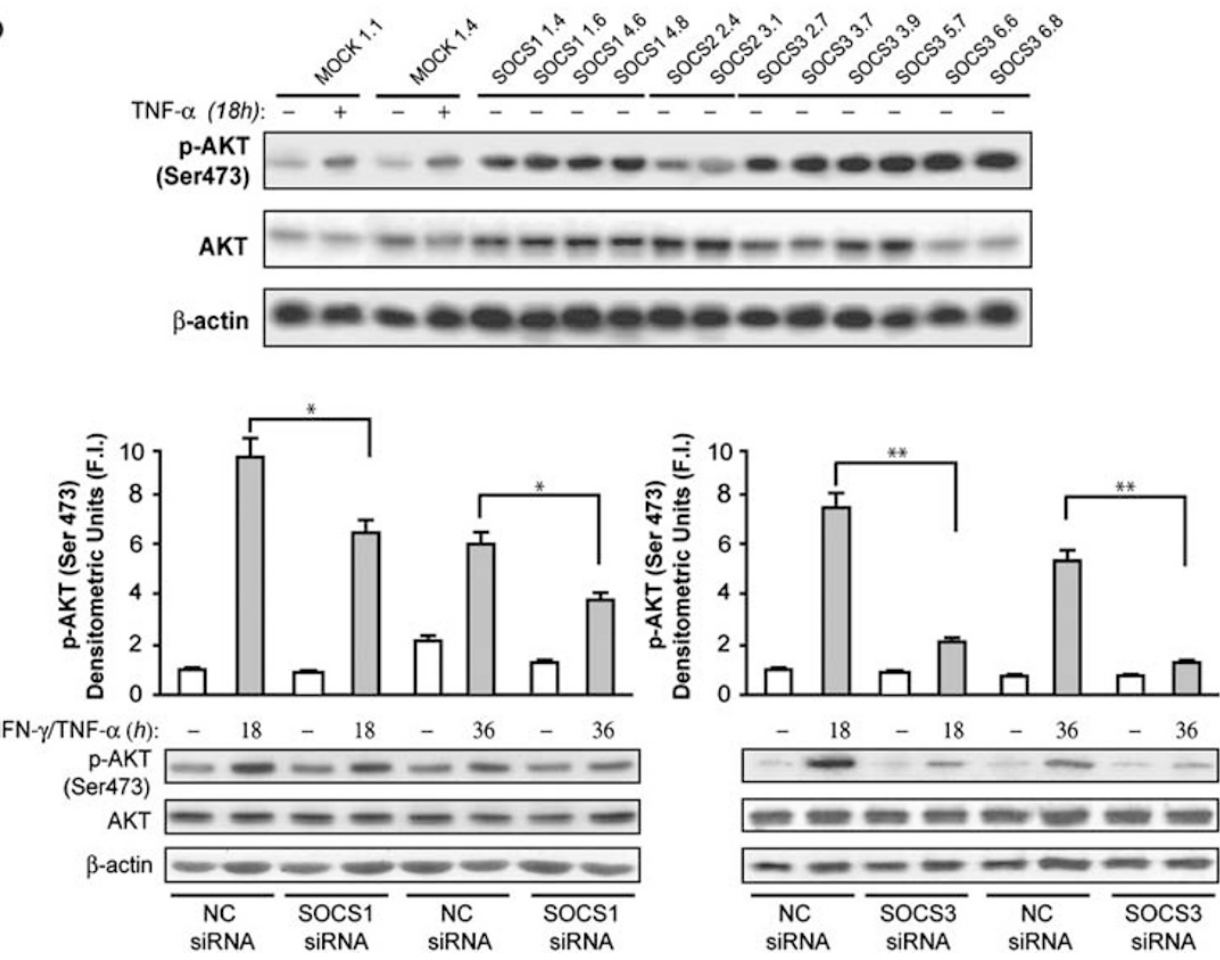

Figure 3 SOCS3 and SOCS1 participate to the induction of RAS activation and AKT phosphorylation in cytokine-activated keratinocytes. (a) RAS activity was measured on lysates obtained from MOCK $(n=2)$, SOCS1 $(n=4)$, SOCS2 $(n=2)$ and SOCS3 $(n=6)$ keratinocyte clones treated with TNF- $\alpha$ for $3 h$. GTP-bound RAS was pulleddown using Raf-1 RBD agarose as substrate and detected by WB with an anti-RAS Ab. RAS activity was also analyzed in SOCS1-or SOCS3-interfered keratinocytes treated with IFN- $\gamma / T N F-\alpha$ for 12 or $24 \mathrm{~h}$ and compared with that observed in NC-transfected cells. Data are expressed as F.I. \pm S.D. between IFN- $\gamma / T N F-\alpha$ time-course points and untreated samples (time 0 ), to which a value of 1 were given. (b) Phosphorylation of AKT in Ser 473 was evaluated by immunoblotting on protein lysates obtained from MOCK and SOCS clones treated with TNF- $\alpha$ for $6 \mathrm{~h}$, and from SOCS1-or SOCS3-silenced keratinocytes stimulated with IFN- $\gamma / T N F-\alpha$ for 18 or $36 \mathrm{~h}$. Filters were stripped and re-probed with an anti-AKT Ab. In RNA interference experiments, F.I. values relative to SOCS1 or SOCS3-silenced keratinocytes were compared with those obtained from NC-interfered cells. In RNA interference experiments, data were obtained by three independent experiments. Values of $P<0.05\left({ }^{*}\right)$ and $P<0.01\left({ }^{*}\right)$ were considered significant

TNF- $\alpha$ treatments was significantly lower than that of healthy strains ( $>$ three times decrement in psoriatic cells, $P<0.01$ ) (Figure 7a). However, when SOCS3 or SOCS1 mRNA were silenced by transient treatment of cultures with specific
siRNA, psoriatic keratinocytes strains became more susceptible to apoptosis induced by IFN- $\gamma$ and TNF- $\alpha$ treatments (Figure $7 \mathrm{~b}$ ). Similar effects were observed when cultured psoriatic keratinocytes were treated with increasing doses of 



b
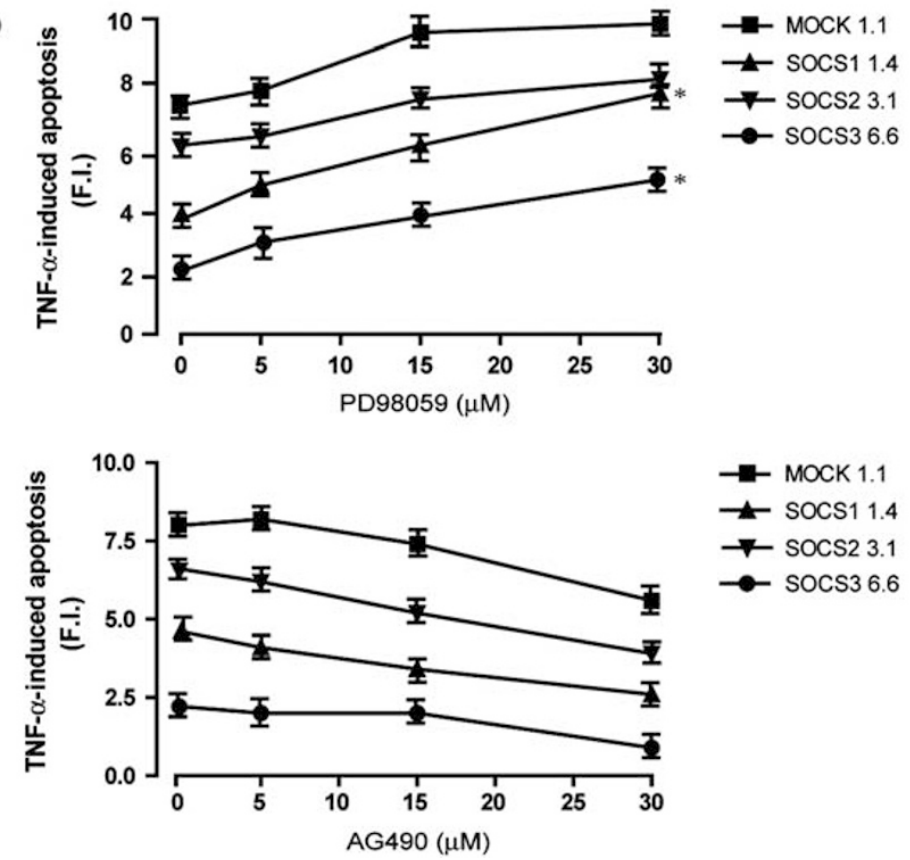

Figure 4 Inhibition of PI3K/AKT pathway, but not of JAK2, in SOCS1 and SOCS3 clones increases their sensitivity to TNF- $\alpha$-induced apoptosis. (a) SOCS1 ( $\mathbf{\Delta}, n=6)$ and $\operatorname{SOCS3}(\bullet, n=6)$ clones, together with $\operatorname{MOCK}(\boldsymbol{\square}, n=6)$ and $\operatorname{SOCS2}(\boldsymbol{\nabla}, n=6)$ clones used as control, were pre-incubated or not with increasing doses $(3$ and $15 \mu \mathrm{M}$ final concentration) of the chemical PI3K inhibitor, Ly294002, and then left untreated or stimulated with TNF- $\alpha$ for $48 \mathrm{~h}$. (b) Similarly, MOCK 1.1, SOCS1 1.4, SOCS2 3.1 and SOCS3 6.6 clones were pre-treated or not with increasing doses (from 5 to $30 \mu \mathrm{M}$ ) of PD98059 (ERK1/2 inhibitor) or AG490 (JAK2 inhibitor), and then stimulated with TNF- $\alpha$. In all experiments, apoptosis was evaluated by measuring Annexin/PI fluorescent staining. Data are expressed as F.I. between TNF- $\alpha$-treated cells and untreated samples, to which a value of 1 was given. Values of $P<0.05\left(^{*}\right)$ and $P<0.01\left(^{* *}\right)$ were considered significant

Ly29 (Figure 7c). Although the chemical inhibition of PI3K/ AKT signaling enhanced apoptosis in both healthy and psoriatic cells, this effect was more evident in psoriatic strains (Figure 7c; $P<0.05$ and $P<0.01$ for healthy and psoriatic, respectively), suggesting a functional link between $\mathrm{PISK} / \mathrm{AKT}$ pathway, sustained by SOCS1 and SOCS3, and the peculiar resistance of psoriatic keratinocytes to cytokineinduced apoptosis.

\section{Discussion}

Psoriasis is an immune-mediated skin disease driven by memory-effector, skin-homing $T$ cells. In psoriatic skin lesions, $T$ cells locally release pro-inflammatory cytokines, such as IFN- $\gamma$ and TNF- $\alpha$, responsible for the activation of the resident epidermal keratinocytes skin. ${ }^{26,30}$ Because of their intrinsically altered capacity to respond to $\mathrm{IFN}-\gamma$ and 


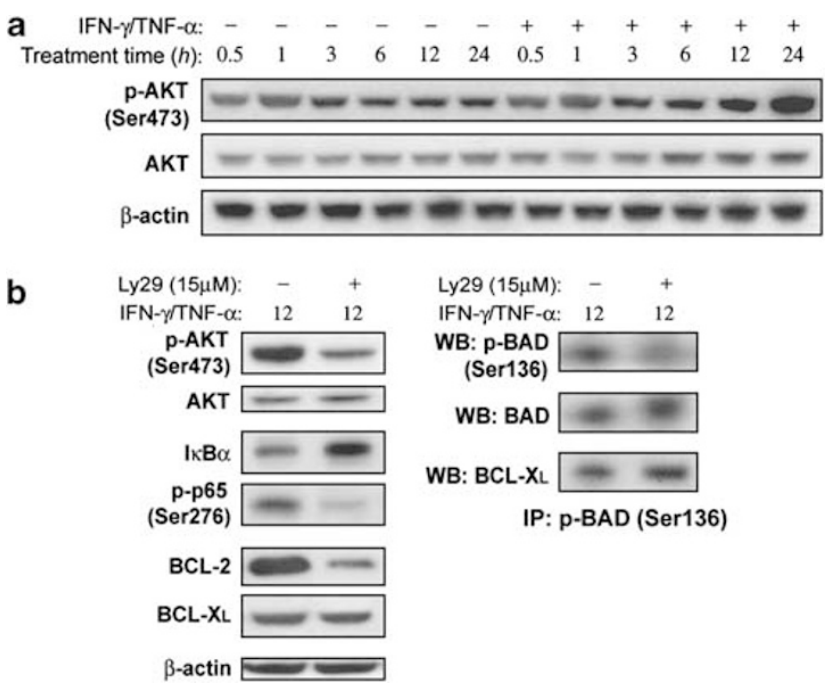

Figure 5 AKT phosphorylation determines the activation of NF- $\kappa B$ cascade and, in parallel, the phosphorylation and inhibition of BAD molecule. (a) AKT phosphorylation (Ser 473) was monitored in keratinocytes treated with IFN- $\gamma /$ TNF- $\alpha$ at different time points by WB analysis. Filters were stripped and re-probed with an anti-AKT Ab. (b) Primary human keratinocytes were pre-treated with Ly294002 $(15 \mu \mathrm{M})$ for $2 \mathrm{~h}$ and then stimulated with IFN- $\gamma / \mathrm{TNF}-\alpha$ for $12 \mathrm{~h}$. In left panel, protein extracts were subjected to WB with anti-phospho AKT (Ser473), anti-AKT, antiI $\kappa \mathrm{B} \alpha$, anti-phospho 065 (Ser 276), anti-BCL-2, anti-BCL- $\mathrm{X}_{\mathrm{L}}$ and anti- $\beta$-actin Abs. In right panels, co-immunoprecipitation of endogenous phospho-BAD (Ser136) was followed by WB using anti-BCL- $X_{L}$ Ab. Filter was stripped and re-probed with BAD Ab

TNF- $\alpha$, psoriatic keratinocytes express a plethora of inflammatory mediators, involved in the chronicization of the local immune responses. ${ }^{26,31}$ Additionally, psoriatic keratinocytes exhibit a marked hyper-proliferation and an incomplete terminal differentiation in response to mitogenic signals and are characterized by an aberrant resistance to apoptosis, which synergistically contribute to the peculiar epidermal thickening of diseased skin. ${ }^{18,26,32,33}$ Although many efforts have been made to elucidate the mechanisms responsible for the alterations in psoriatic epidermis of proliferation and differentiation programs, ${ }^{34,35}$ to date limited information exist on the processes concurring to the reduced susceptibility of affected keratinocytes to cytokine-induced apoptosis. In this study, we have investigated on the involvement of SOCS3 and SOCS1 molecules in protecting psoriatic keratinocytes from IFN- $\gamma / T N F-\alpha$-induced cell death. This interest arose from previous observations that SOCS1 has been described as an anti-apoptotic molecules, and that psoriatic keratinocytes express enhanced levels of SOCS1 compared with healthy cells. ${ }^{6,12,13}$ We extended our analysis to SOCS3 molecule, whose expression is highly induced by IFN- $\gamma$ and TNF- $\alpha$, unveiling that activated psoriatic keratinocyte cultures have a particular attitude to upregulate SOCS3, other than SOCS1, as compared with healthy cells. In fact, SOCS3 highly accumulated in vivo in psoriasis plaques, but, differently from SOCS1 mainly detected in the basal and spinosum layers of epidermis, ${ }^{6}$ it abundantly localized in the upper stratum of the epidermis. The abnormal SOCS1 expression in psoriatic keratinocytes depended on an alteration of the transcriptional machinery regulating its promoter function, involving growth factor independence- $1 \mathrm{~b}$ and Kruppel-like factor 4 transcription factors. ${ }^{6}$ Aimed at correlating the high SOCS3 and SOCS1 expression to the resistance to apoptosis of psoriatic keratinocytes, we manipulated their expression and evaluated the effects on cytokine-induced cell death. In both experimental conditions, SOCS1 and, more markedly, SOCS3 could suppress the IFN- $\gamma / \mathrm{TNF}-\alpha$-induced apoptosis in human keratinocytes. Although we show here for the first time that SOCS3 and SOCS1 confer resistance to cytokine-induced apoptosis to keratinocytes, this SOCS function has been described in the past for other cell types, including fibroblasts and pancreatic cells. ${ }^{12-14}$ In these cell systems, the mechanisms underlying the SOCS1 and SOCS3 inhibition of apoptosis involved the activation of RAS-dependent ERK1/ 2, p38 and JNK signalings. ${ }^{12,14}$ In our study, we could demonstrate that SOCS1 and, at a higher extent, SOCS3, transiently induced by IFN- $\gamma$ plus TNF- $\alpha$, could protect keratinocytes from apoptosis by sustaining another RASdependent pathway, in particular the PI3K/AKT cascade. This pathway specifically protected keratinocytes from apoptosis, since the inhibition of JAK2 activity in SOCS1 and SOCS3 keratinocyte clones did not affect their apoptotic rates. The slight increase of TNF- $\alpha$-induced apoptosis determined by ERK1/2 inhibitor PD98059 in SOCS1 and SOCS3 clones confirmed the protective and pro-survival role of ERK1/2 in human keratinocytes. ${ }^{5}$ However, differently from ERK $1 / 2$ cascade, linked to the regulation of anti-inflammatory and proliferative programs following cytokine exposure, PI3K/AKT pathway seems to be implicated in mechanisms aimed at rescuing keratinocytes from apoptosis. This has been demonstrated in keratinocytes exposed to UV, which show reduced apoptosis subsequently to a downregulation of the intracellular pro-apoptotic factors $\mathrm{BAD}, \mathrm{IKB} \alpha$ and GSK3. ${ }^{23,24,36}$

Concerning the AKT downstream effects in keratinocytes, we found that this anti-apoptotic pathway determined the activation of NF- $\kappa \mathrm{B}$ and, in parallel, the inhibition of the proapoptotic function of BAD. It is known that NF- $\kappa$ B pathway protects a number of cell types from apoptosis by inducing the expression of anti-apoptotic proteins, including survivin, FLICE-like inhibitory protein and members of the inhibitor of apoptosis family, such as BCL-2 molecule. ${ }^{37-39}$ Accordingly to these studies, we demonstrated that $\mathrm{BCL}-2$ expression is positively regulated by PI3K-AKT pathway, possibly through a direct action of NF- $\kappa$ B induced by pro-inflammatory cytokines in human keratinocytes.

In relation to the role of $B A D$ molecule, it has been reported that in UVB-irradiated keratinocytes, AKT-induced phosphorylation of $B A D$ determines its inactivation and its release from $\mathrm{BCL}-\mathrm{X}_{\mathrm{L}} \cdot{ }^{24}$ This event results in the suppression of proapoptotic mechanisms, by directly interfering with BAX/BAKinduced mitochondrial permeabilization. ${ }^{24}$ In light of this knowledge, it is reasonable to suppose that NF- $\kappa$ B activation and BAD inhibition contribute to re-establishment of survival and protective programs also when apoptosis is induced by pro-inflammatory cytokines.

Interestingly, the expression of phosphorylated AKT was intense in lesional psoriatic skin, and paralleled the presence of an abundant $\mathrm{CD}^{+}{ }^{+} \mathrm{T}$-cell infiltrate. Contrarily, healthy and 
a

p-AKT

(Ser473)
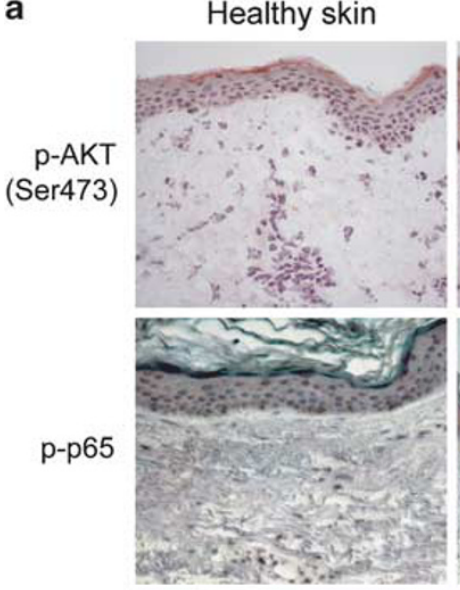

p-BAD

(Ser136)
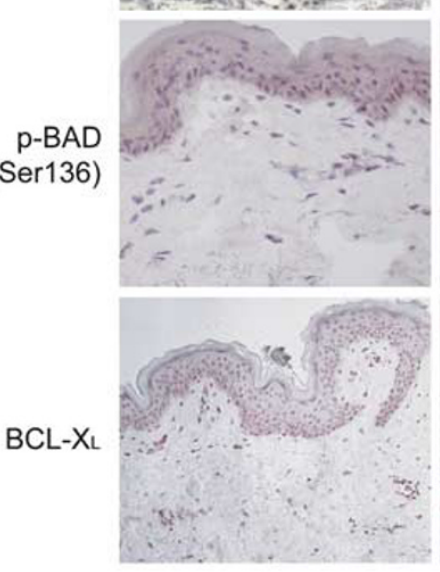

NLS Psoriatic
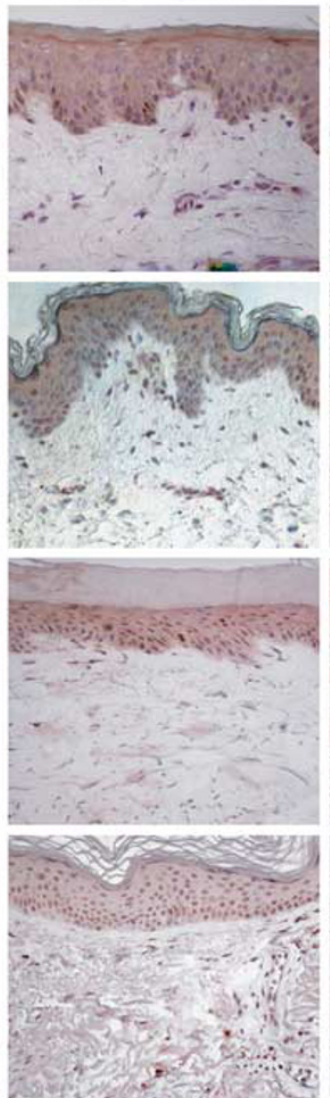

LS Psoriatic
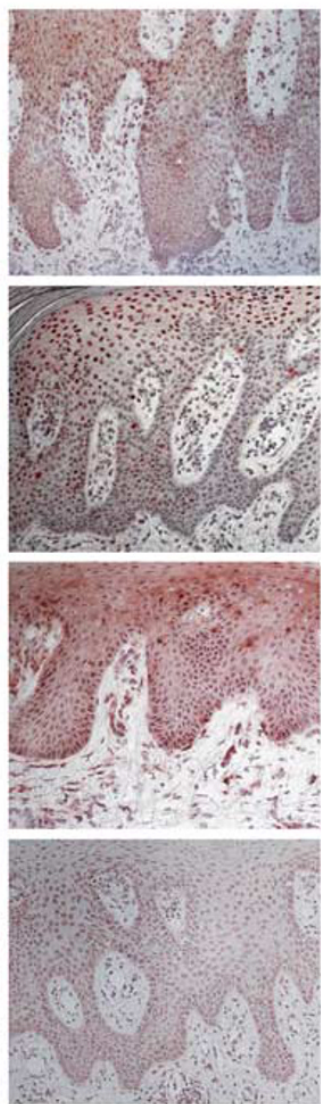

b

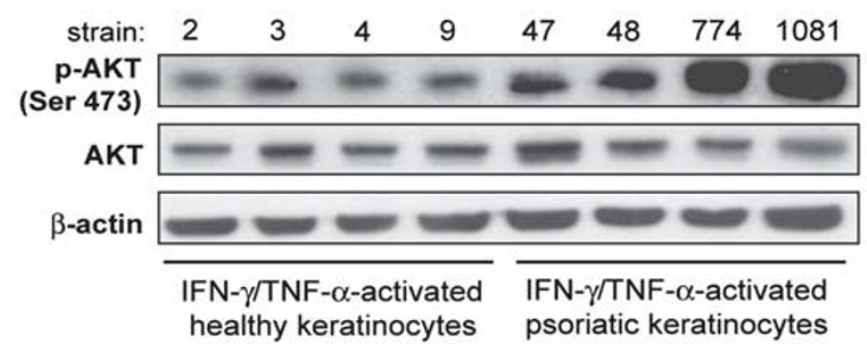

Figure 6 Expression of phospho-AKT, phospho-p65, phospho-BAD and BCL- $X_{L}$ is upregulated in activated psoriatic keratinocytes. (a) Immunohistochemistry for p-AKT (Ser473) and p-BAD (Ser136) were performed on frozen sections from biopsies of healthy $(n=4)$ and psoriatic $(n=4)$ skin, including NLS and LS areas of plaques. Phosphop65 and BCL- $X_{L}$ in vivo staining was performed on paraffin-embedded sections of healthy, NL and LS skin biopsies. Sections were counterstained with Mayer's H\&E. One out of four representative staining of psoriatic skin is shown. (b) Phospho-AKT (Ser473) level was detected in protein lysates of cultured healthy and psoriatic keratinocytes treated with IFN- $\gamma / \mathrm{TNF}-\alpha$ for $12 \mathrm{~h}$. Filter was stripped and re-probed with anti-AKT Ab. One out of six representative healthy and psoriatic keratinocyte strains is shown

NLS psoriatic skin exhibited a similar expression of phosphorylated AKT, indicating that the enhanced AKT expression in lesional plaques was the consequence of the microenviromental cytokine milieu. However, in vitro activation of AKT was more evident in psoriatic keratinocyte strains compared with the healthy ones, indicating again that psoriatic cells are more responsive to cytokine stimulations. Consistently with AKT in vivo distribution, the expression of phospho-BAD and $B C L-X_{L}$ in psoriatic skin was intense and accumulated in the upper layers of psoriatic epidermis. The findings that SOCS3 co-localized with phospho-AKT, phospho-BAD and BCL- $\mathrm{X}_{\mathrm{L}}$ molecules in the same epidermal compartments suggest their possible cross regulation in vivo. Interestingly, together with other apoptosis-related proteins, BCL-2 has also been detected in the epidermis of skin affected by psoriasis, and found to be reduced in lesional keratinocytes after treatment with TNF- $\alpha$-targeting agents. ${ }^{25}$

The evidence that SOCS1 and SOCS3 render psoriatic keratinocytes resistant to apoptosis comes from SOCS1 and SOCS3 mRNA knocking-down experiments in these cell strains, where the IFN- $\gamma$ plus TNF- $\alpha$-induced apoptosis were efficiently inhibited. The chemical inhibition of PI3K/AKT cascade also reverted the capability of psoriatic keratinocytes to resist to pro-apoptotic stimuli. Further experiments aimed at rescuing the resistance to IFN- $\gamma / \mathrm{TNF}-\alpha$-induced death through the ectopic overexpression of AKT gene in SOCS1- or 



Figure 7 SOCS1 and SOCS3 depletion, as well as the chemical inhibition of $\mathrm{PI}$ IK/AKT axis, in activated psoriatic keratinocytes revert their resistance to IFN- $\gamma /$ TNF- $\alpha$-induced cell death. (a) Cultured healthy $(\square, n=6)$ and psoriatic $(\triangle, n=6)$ keratinocytes were left untreated or treated with IFN- $\gamma / T N F-\alpha$ for $48 \mathrm{~h}$. (b) Cultured psoriatic keratinocytes $(n=6)$ were transfected with increasing doses of SOCS1 plus SOCS3 siRNA $(\triangle)$ or irrelevant siRNA $(\mathbf{\Delta})(20$ and $80 \mathrm{nM})$, then left untreated or treated with IFN- $\gamma /$ TNF- $\alpha$ for $48 \mathrm{~h}$. (c) Cultured healthy $(\square, n=6)$ and psoriatic $(\triangle, n=6)$ keratinocytes were pre-incubated with increasing doses of Ly294002 $(15$ and $25 \mu \mathrm{M})$ for $2 \mathrm{~h}$ and then left untreated or treated with IFN- $\gamma / T N F-\alpha$ for $48 \mathrm{~h}$. In all experiments, apoptosis was evaluated by measuring Annexin/PI fluorescent staining. Data were expressed as ratio between TNF- $\alpha$-treated cells and untreated samples. Values of $P<0.05\left(^{*}\right)$ and $P<0.01\left(^{* *}\right)$ were considered significant
SOCS3-depleted psoriatic keratinocytes will definitely confirm the functional link between SOCS1 and SOCS3 and AKTmediated apoptosis, typically impaired in psoriatic cells.

In conclusion, this study demonstrated that the strong upregulation of SOCS1 and SOCS3 in activated psoriatic keratinocytes concurs in reducing apoptosis induced by proinflammatory cytokines in these cells. The higher SOCS1 and SOCS3 expression in psoriatic keratinocytes compared with healthy cells likely could represent a mechanism by which psoriatic cells protect themselves from exaggerated and repeated stimulation by IFN- $\gamma$ and TNF- $\alpha$ (Supplementary Figure) occurring in active skin lesions. On the other hand, the enhanced SOCS1 and SOCS3 expression could have side effects, concurring at reducing susceptibility to IFN- $\gamma / T N F-\alpha-$ effects, expressed not only in terms of pro-inflammatory gene expression but also of apoptosis induction. The enhanced SOCS1 and SOCS3 expression in psoriatic keratinocytes could ultimately contribute to the expression of the peculiar epidermal thickening of psoriatic skin (Supplementary Figure).

In light of our findings, the use of inhibitors of PI3K/AKT axis in epidermal keratinocytes may be therapeutically relevant for the treatment of psoriasis, but also for other skin diseases characterized by a deficient keratinocyte apoptosis, including non-melanoma skin cancers. ${ }^{40}$ Further studies using chemical AKT inhibitors in experimental models of psoriasis in vitro and in vivo should better define the role of the anti-apoptotic programs in the psoriatic epidermal thickening.

\section{Materials and Methods}

Subjects. Ten patients with severe chronic plaque psoriasis were included in this study. Among these patients, four were selected for immunohistochemical studies and six to establish keratinocyte cultures. Patients had definite psoriasis diagnosed according to standard criteria, and they had not received any systemic or topical therapy for at least 1 month before skin donation. Skin was also obtained from ten healthy subjects undergoing plastic surgery.

Keratinocyte cultures and treatments. Normal human keratinocytes were obtained from skin biopsies of healthy volunteers or psoriatic patients and cultured, as previously reported. ${ }^{5,6}$ Stimulations with $200 \mathrm{U} / \mathrm{ml}$ human recombinant IFN- $\gamma$ (R\&D Systems, Minneapolis, MN, USA) and $50 \mathrm{ng} / \mathrm{ml}$ human recombinant TNF- $\alpha$, as well as treatments with the chemical Ly294002 or MG132 inhibitors (Calbiochem, Gibbstown, NJ, USA), were performed in keratinocyte basal medium (Clonetics, Walkersville, MD, USA). The HaCaT human keratinocyte cell line (NE Fusenig, Deutsches Krebsforschungszentrum, Heidelberg, Germany) was grown and stimulated as previously described. ${ }^{4,5}$

Permanent transfections. HaCaT cells were stably transfected with myc/SOCS1, myc/SOCS2, myc/SOCS3 or empty pcDNA3 plasmids as previously reported. ${ }^{4,5}$ When indicated, $\mathrm{HaCaT}$ clones were treated with the chemical inhibitors Ly29, PD98059 or AG490 (Calbiochem) in DMEM.

RNA Isolation and Real time RT-PCR. Total RNA isolation and real-time RT-PCR analyses for SOCS1 and SOCS3 mRNA expression were performed as previously described. ${ }^{5,6}$ Fluorescence intensity was analyzed by the ABI PRISM SDS 7000 PCR Instrument (Applied Biosystems, Branchburg, NJ, USA). The foldinduction value for triplicate wells was averaged, and data were presented as the mean \pm S.D.

Immunoprecipitation, immunoblotting and densitometry. Protein extract preparation, immunoprecipitation and immunoblotting were performed accordingly to standard procedures. ${ }^{5,6}$ The Abs used for the study were as follows: anti-SOCS1 and anti-SOCS3 (MBL International Corporation, Nakaku Nagoya, Japan), anti-phospho-p65 (Ser276), anti- $\kappa \mathrm{B} \alpha$, HRP-conjugated anti-c-myc (9E10), anti- $\beta$-actin (all from Santa Cruz Biotechnology, Santa Cruz, CA, USA) 
and anti-phospho-AKT (Ser473), anti-AKT, anti-phospho-BAD (Ser136) and antiBAD (all from Cell Signaling, Danvers, MA, USA). Anti-BCL- $X_{L}$ was purchased from Calbiochem (La Jolla, CA, USA). Immunoblots of experiments evaluating phospho-AKT and GTP-RAS levels were subjected to densitometry using an Imaging Densitometer model GS-670 (Bio-Rad, Hercules, CA, USA) supported by the Molecular Analyst software (Bio-Rad), and band intensities were evaluated in three independent experiments. Data are expressed as fold-induction (F.I.) \pm S.D. in experimental IFN- $\gamma / \mathrm{TNF}-\alpha$ time-course relative to untreated samples, to which were given a value of 1 .

Transient RNA interference. SOCS1 or SOCS3 were knocked down in keratinocyte cultures as previously described. ${ }^{5,6}$ SOCS1 (L-011511-00-0005), SOCS3 (L-004299-00-0005) or irrelevant (L-011511-00-0005) pool of four small siRNA (Dharmacon RNA Technology, Lafayette, CO, USA) were used at a final concentration ranging from 20 to $80 \mathrm{nM}$. After $24 \mathrm{~h}$ of transfection, cells were stimulated with IFN- $\gamma$ and TNF- $\alpha$ for the indicated time periods.

In vitro kinase assays. RAS activation assays was performed using commercial kit from Upstate (Upstate Biotechnologies, Temecula, CA, USA), according to manufacturer' instructions.

Apoptosis analysis. Apoptosis of keratinocytes was evaluated using the Genzyme TACS Annexin V apoptosis detection kit (R\&D Systems) or using the carboxyfluorescein FLICA assay kit (B-Bridge International, Sunnyvale, CA, USA). Viable, necrotic and apoptotic were analyzed by flow cytometry. Cells were analyzed with a FACScan equipped with Cell Quest software (Becton Dickinson, Mountain View, CA, USA). Data were expressed as F.I. between cytokine-treated and untreated samples, to which a value of 1 was given.

Immunohistochemistry. Paraffin-embedded sections or cryostatic sections were obtained from biopsies of psoriatic skin including LS, Pre-LS and NLS zones of evolving plaques as well as healthy skin. Paraffin-embedded sections were incubated with Abs against BCL- $X_{L}$ (Calbiochem) or phospho-p65 (Cell Signaling). Cryostatic sections were incubated with anti-SOCS3 (MBL), -CD3 (BD), -p-AKT (Cell Signaling) and -p-BAD (Cell Signaling) Abs. Secondary biotinylated mAbs and staining kits (Vector Laboratories, Burlinagame, CA, USA) were used to develop immunoreactivities.

Statistical analysis. Statistical significance was evaluated using Wilcoxon's signed rank test (SigmaStat; Jandel, San Rafael, CA, USA). Values of $P \leq 0.05$ were considered significant.

\section{Conflict of Interest}

The authors declare no conflict of interest.

Acknowledgements. We thank Dr. Rosanna Sestito who carried out SOCS3 IHC analyses. This work was supported by the Italian Ministry of Health.

1. Yoshimura A, Naka T, Kubo M. SOCS proteins, cytokine signalling and immune regulation. Nat Rev Immunol 2007; 7: 454-465.

2. Dimitriou ID, Clemenza L, Scotter AJ, Chen G, Guerra FM, Rottapel R. Putting out the fire: coordinated suppression of the innate and adaptive immune systems by SOCS1 and SOCS3 proteins. Immunol Rev 2008; 224: 265-283.

3. Ramana CV, Gil MP, Schreiber RD, Stark GR. Stat1-dependent and -independent pathways in IFN-gamma-dependent signaling. Trends Immunol 2002; 23: 96-101.

4. Federici M, Giustizieri ML, Scarponi C, Girolomoni G, Albanesi C. Impaired IFN-gammadependent inflammatory responses in human keratinocytes overexpressing the suppressor of cytokine signaling 1. J Immunol 2002; 169: 434-442.

5. Madonna S, Scarponi C, De Pita' O, Albanesi C. Suppressor of cytokine signaling 1 inhibits IFN-gamma inflammatory signaling in human keratinocytes by sustaining ERK1/2 activation. FASEB J 2008; 22: 3287-3297.

6. Madonna S, Scarponi C, Sestito R, Pallotta S, Cavani A, Albanesi C. The IFN-gammadependent suppressor of cytokine signaling 1 promoter activity is positively regulated by IFN regulatory factor-1 and Sp1 but repressed by growth factor independence-1b and Krüppel-like factor-4, and it is dysregulated in psoriatic keratinocytes. $\mathrm{J} / \mathrm{mm}$ uno/ 2010; 185: 2467-2481.
7. Doti N, Scognamiglio PL, Madonna S, Scarponi C, Ruvo M, Perretta G et al. New mimetic peptides of Kinase Inhibitory Region (KIR) of SOCS1 through focused peptide libraries. Biochem J 2012; 443: 231-240.

8. Strebovsky J, Walker P, Lang R, Dalpke AH. Suppressor of cytokine signaling 1 (SOCS1) limits NF kappa B signaling by decreasing p65 stability within the cell nucleus. FASEB J 2011; 25: 863-874.

9. He Y, Zhang W, Zhang R, Zhang H, Min W. SOCS1 inhibits tumor necrosis factor-induced activation of ASK1-JNK inflammatory signaling by mediating ASK1 degradation. $J$ Biol Chem 2006; 281: 5559-5566.

10. Croker BA, Kiu H, Pellegrini M, Toe J, Preston S, Metcalf D et al. IL-6 promotes acute and chronic inflammatory disease in the absence of SOCS3. Immunol Cell Biol 2011; 26: 1-6.

11. van de Geijn GJ, Gits J, Touw IP. Distinct activities of suppressor of cytokine signaling (SOCS) proteins and involvement of the SOCS box in controlling G-CSF signaling. $J$ Leukoc Biol 2004; 76: 237-244.

12. Morita $Y$, Naka T, Kawazoe $Y$, Fujimoto M, Narazaki M, Nakagawa $R$ et al. Signals transducers and activators of transcription (STAT)-induced STAT inhibitor-1 (SSI-1)/ suppressor of cytokine signaling-1 (SOCS-1) suppresses tumor necrosis factor alpha-induced cell death in fibroblasts. Proc Natl Acad Sci USA 2000; 97: 5405-5410.

13. Kimura A, Naka T, Nagata S, Kawase I, Kishimoto T. SOCS-1 suppresses TNF-alpha-induced apoptosis through the regulation of Jak activation. Int Immunol 2004; 16: 991-999.

14. Bruun C, Heding PE, Rønn SG, Frobøse H, Rhodes CJ, Mandrup-Poulsen T et al. Suppressor of cytokine signalling-3 inhibits Tumor necrosis factor-alpha induced apoptosis and signalling in beta cells. Mol Cell Endocrinol 2009; 311: 32-38.

15. Tomita S, Ishibashi K, Hashimoto K, Sugino T, Yanagida T, Kushida N et al. Suppression of SOCS3 increases susceptibility of renal cell carcinoma to interferon- $\alpha$. Cancer Sci 2011; 102: $57-63$.

16. Lippens $\mathrm{S}$, Hoste E, Vandenabeele $\mathrm{P}$, Agostinis $\mathrm{P}$, Declercq W. Cell death in the skin. Apoptosis 2009; 14: 549-569

17. Reefman E, Limburg PC, Kallenberg CG, Bijl M. Apoptosis in human skin: role in pathogenesis of various diseases and relevance for therapy. Ann NY Acad Sci 2005; 1051: 52-63.

18. Raj D, Brash DE, Grossman D. Keratinocyte apoptosis in epidermal development and disease. J Invest Dermatol 2006; 126: 243-257.

19. Dallaglio K, Marconi A, Pincelli C. Survivin: a dual player in healthy and diseased skin. J Invest Dermatol 2012; 132: 18-27.

20. Fukuya $Y$, Higaki M, Higaki $Y$, Kawashima M. Effect of vitamin D3 on the increased expression of $\mathrm{Bcl}-\mathrm{xL}$ in psoriasis. Arch Dermatol Res 2002; 293: 620-625.

21. Györffy B, Schäfer R. Biomarkers downstream of RAS: a search for robust transcriptional targets. Curr Cancer Drug Targets 2010; 10: 858-868.

22. Espada J, Galaz S, Sanz-Rodríguez F, Blázquez-Castro A, Stockert JC, Bagazgoitia L et al. Oncogenic H-Ras and PI3K signaling can inhibit E-cadherin-dependent apoptosis and promote cell survival after photodynamic therapy in mouse keratinocytes. $J$ Cell Physiol 2009; 219: 84-93.

23. Kim NH, Jeon S, Lee HJ, Lee AY. Impaired PI3K/Akt activation-mediated NF-kappaB inactivation under elevated TNF-alpha is more vulnerable to apoptosis in vitiliginous keratinocytes. J Invest Dermatol 2007; 127: 2612-2617.

24. Claerhout S, Decraene D, Van Laethem A, Van Kelst S, Agostinis P, Garmyn M. AKT delays the early-activated apoptotic pathway in UVB-irradiated keratinocytes via BAD translocation. J Invest Dermatol 2007; 127: 429-438.

25. Kokolakis G, Giannikaki E, Stathopoulos E, Avramidis G, Tosca AD, Krüger-Krasagakis S. Infliximab restores the balance between pro- and anti-apoptotic proteins in regressing psoriatic lesions. Br J Dermatol 2012; 166: 491-497.

26. Albanesi C, Pastore S. Pathobiology of chronic inflammatory skin diseases: interplay between keratinocytes and immune cells as a target for anti-inflammatory drugs. Curr Drug Metab 2010; 11: 210-227.

27. Doger FK, Dikicioglu E, Ergin F, Unal E, Sendur N, Uslu M. Nature of cell kinetics in psoriatic epidermis. J Cutan Pathol 2007; 34: 257-263.

28. Wrone-Smith T, Mitra RS, Thompson CB, Jasty R, Castle VP, Nickoloff BJ. Keratinocytes derived from psoriatic plaques are resistant to apoptosis compared with normal skin. Am J Pathol 1997; 151: 1321-1329.

29. Wrone-Smith T, Johnson T, Nelson B, Boise LH, Thompson CB, Núñez G et al. Discordant expression of $\mathrm{Bcl}-\mathrm{x}$ and $\mathrm{Bcl}-2$ by keratinocytes in vitro and psoriatic keratinocytes in vivo. Am J Pathol 1995; 146: 1079-1088.

30. Di Meglio P, Perera GK, Nestle FO. The multitasking organ: recent insights into skin immune function. Immunity 2011; 35: 857-869.

31. Albanesi C, De Pità O, Girolomoni G. Resident skin cells in psoriasis: a special look at the pathogenetic functions of keratinocytes. Clin Dermatol 2007; 25: 581-588.

32. Zheng Y, Danilenko D M, Valdez P, Kasman I, Eastham- Anderson J, Wu J et al. Interleukin-22, a $\mathrm{T}(\mathrm{H}) 17$ cytokine, mediates IL-23-induced dermal inflammation and acanthosis. Nature 2007; 445: 648-651.

33. Qin JZ, Chaturvedi V, Denning MF, Choubey D, Diaz MO, Nickoloff BJ. Role of NF-kappaB in the apoptotic-resistant phenotype of keratinocytes. J Biol Chem 1999; 274: 37957-37964.

34. Wolk K, Haugen HS, Xu W, Witte E, Waggie K, Anderson M et al. IL-22 and IL-20 are key mediators of the epidermal alterations in psoriasis while IL-17 and IFN-gamma are not. $J$ Mol Med 2009; 87: 523-536. 
35. Eyerich S, Eyerich K, Pennino D, Carbone T, Nasorri F, Pallotta S et al. Th22 cells represent a distinct human $\mathrm{T}$ cell subset involved in epidermal immunity and remodeling J Clin Invest 2009; 119: 3573-3585.

36. Schieke SM, von Montfort C, Buchczyk DP, Timmer A, Grether-Beck S, Krutmann J et al. Singlet oxygen-induced attenuation of growth factor signaling: possible role of ceramides. Free Radic Res 2004; 38: 729-737.

37. Lin J, Guan Z, Wang C, Feng L, Zheng Y, Caicedo E et al. Inhibitor of differentiation 1 contributes to head and neck squamous cell carcinoma survival via the NF-kappaB/survivin and phosphoinositide 3-kinase/Akt signaling pathways. Clin Cancer Res 2010; 16 77-87.

38. Bai S, Liu H, Chen KH, Eksarko P, Perlman H, Moore TL et al. NF-kappaB-regulated expression of cellular FLIP protects rheumatoid arthritis synovial fibroblasts from tumo necrosis factor alpha-mediated apoptosis. Arthritis Rheum 2004; 50: 3844-3855.
39. Kwon HK, Hwang JS, So JS, Lee CG, Sahoo A, Ryu JH et al. Cinnamon extract induces tumor cell death through inhibition of NFkappaB and AP1. BMC Cancer 2010; 10: 392-402.

40. Samuels Y, Ericson K. Oncogenic PI3K and its role in cancer. Curr Opin Oncol 2006; 18: 77-82.

(c)

Cell Death and Disease is an open-access journal published by Nature Publishing Group. This work is licensed under the Creative Commons Attribution-NonCommercial-No Derivative Works 3.0 Unported License. To view a copy of this license, visit http://creativecommons.org/licenses/by-nc-nd/3.0/

Supplementary Information accompanies the paper on Cell Death and Disease website (http://www.nature.com/cddis) 